\title{
The Quantum Ising Chain with a Generalized Defect
}

\author{
Uwe Grimm \\ Physikalisches Institut der Universität Bonn \\ Nußallee 12, 5300 Bonn 1, Germany \\ Preprint BONN-HE-89-15 (November 1989) \\ PACS numbers: $05.50 .+q, 75.10 . J m$
}

\begin{abstract}
The finite-size scaling properties of the quantum Ising chain with different types of generalized defects are studied. These not only mean an alteration of the coupling constant as previously examined, but an additional arbitrary transformation in the algebra of observables at one site of the chain. One can distinguish between two classes of generalized defects: those which do not affect the finite-size integrability of the Ising chain, and on the other hand those that destroy this property. In this context, finite-size integrability is always understood as a synonym for the possibility to write the Hamiltonian of the finite chain as a bilinear expression in fermionic operators by means of a Jordan-Wigner transformation. Concerning the first type of defect, an exact solution for the scaling spectrum is obtained for the most universal defect that preserves the global $\mathbf{Z}_{2}$ symmetry of the chain. It is shown that in the continuum limit this yields the same result as for one properly chosen 'ordinary' defect, that is changing the coupling constant only, and thus the finite-size scaling spectra can be described by irreps of a shifted $u(1)$ Kac-Moody algebra. The other type of defect is examined by means of numerical finite-size calculations. In contrast to the first case, these suggest a non-continuous dependence of the scaling dimensions on the defect parameters. A conjecture for the operator content involving only one primary field of a Virasoro algebra with central charge $c=\frac{1}{2}$ is given.
\end{abstract}

(This is a reprint version of a paper that appeared in Nucl. Phys. B 340 (1990) 633-658.) 


\section{Introduction}

The one-dimensional quantum Ising chain, the Hamiltonian limit of the classical two-dimensional Ising model [1] obtained via the transfer-matrix approach (see [2, 3] and references contained therein) belongs to the most frequently investigated and best understood systems in statistical physics. The main cause of this is the fact that the Ising model (without an external magnetic field, however) admits an analytic solution [4] and on top of this undergoes a continuous phase transition at a finite temperature. It is known [5] that at criticality the Ising model describes the field theory of a free massless Majorana fermion, which is a conformal field theory with the central charge $c$ of the Virasoro algebra (VA) being $c=\frac{1}{2}$.

After the importance of the conformal structure [6, 7] 8] of spin chains at the critical point had been recognized, the question arose how defects such that the system stays critical will affect these properties. Clearly one started the investigation of this problem with the simplest example, that is with the Ising quantum chain. The defects in this case were introduced by changing the coupling constant of one nearest-neighbour coupling corresponding to a change of the coupling constant on a half-infinite line in the two-dimensional classical model. The system in this case stays critical 9] and part of the conformal structure is maintained [10, 11, 12, 13.

It is the aim of this article to give a generalization of the case of one localized defect. This is done by performing an arbitrary transformation in the algebra of observables at one site of the chain. In other words, this means that one replaces the Pauli matrix $\sigma^{x}$ in one coupling term of the $N$-site Hamiltonian $H$ of the (ferromagnetic) critical quantum Ising chain with periodic boundary conditions

$$
H=-\frac{1}{2} \sum_{j=1}^{N}\left[\sigma_{j}^{z}+\sigma_{j}^{x} \sigma_{j+1}^{x}\right] \quad, \quad \sigma_{N+1}^{x}=\sigma_{1}^{x},
$$

by a general hermitian $2 \times 2$ matrix, therefore one has four real parameters $e_{1}, e_{x}, e_{y}$, and $e_{z}$ that determine the generalized defect by

$$
\sigma_{N+1}^{x}=e_{1} \mathbf{1}+e_{x} \sigma_{1}^{x}+e_{y} \sigma_{1}^{y}+e_{z} \sigma_{1}^{z},
$$

where 1 denotes the $2 \times 2$ unit matrix and an 'ordinary' defect corresponds to $e_{1}=e_{y}=e_{z}=0$.

The first results for the Ising quantum chain with 'ordinary' defects were obtained by detailed numerical calculation [10, but recently [11, 13] the exact solution for an arbitrary number of isolated defects has been found. The algebraic structure of the spectrum for a fixed number of defects at commensurate distances has been examined in detail in [12 (see also [13]). The results of these investigations for the case of one defect, where the spectrum generating algebra turns out to be a supersymmetric extension (the $\frac{p}{q}=\frac{1}{2}$ higher symmetry algebra [14) of a so-called 'shifted' $u(1)$ Kac-Moody algebra (will be abbreviated by KMA in the sequel), are summarized in the subsequent section. Even more recently, another type of defect (a so-called 'extended' defect), where the coupling constant is altered on a whole fraction of the chain, has been studied [15] and a similar algebraic structure as above has been obtained.

In contrast to the case of an 'ordinary' defect, a generalized defect as defined above might or might not destroy the finite-size integrability property of the chain and one expects a quite different behaviour for various types of generalized defects. Here and in what follows, finite-size integrability always means that one is able to write the Hamiltonian of the finite chain as a bilinear expression in fermionic operators via a Jordan-Wigner transformation [5]. A generic example consisting of a two-parameter family of defects for each of this two classes is examined by analytical respective numerical methods. In the case where the chain stays finite-size integrable the results of the exact solution resemble those of one 'ordinary' defect, which is contained therein for a special choice of the parameters. In particular, the scaling dimensions depend continuously on the parameters of the defects, whereas in the other case the numerical results favour a non-continuous behaviour. The operator content in the latter case is conjectured to be given by a single irrep of a VA with

central charge $c=\frac{1}{2}$ for fixed parameters of the defect. This article restricts to the quantum chain point of view, physical implications for the two-dimensional classical system - if any - are not discussed in this place. 
The paper is organized as follows. In the subsequent section the previously examined boundary conditions and 'ordinary' defects together with the 'generalized' defects (1.2) are introduced more accurately. A short summary of the formerly obtained results is given. This includes a presentation of the character functions of unitary highest weight irreps of the VA with central charge $c=\frac{1}{2}$ and of a shifted $u(1)$ KMA. The generalized defects are then divided into two groups: one type that leaves the quantum chain finite-size integrable and the other that destroys this property.

The third section contains an exact solution for the spectrum of the chain with an finite-size integrable type of defect that maintains the global $\mathbf{Z}_{2}$ symmetry of the Ising chain corresponding to $e_{1}=e_{z}=0$ in Eq. (1.2). This is done employing the methods developed by Lieb, Schultz and Mattis [5] (see also [11, 13]) in a slightly generalized form. The operator content is given in terms of character functions of irreducible representations (irreps) of a shifted $u(1)$ KMA and in fact turns out to have the same form as for one 'ordinary' defect [12, whereas the behaviour on finite lattices appears to be different in general.

In the forth part an example for a two-parameter class of finite-size non-integrable defects that corresponds to taking $e_{1}=e_{y}=0$ in Eq. (1.2) is studied. This can be done through the application of numerical methods only. The effect of this type of defect on the finite-size scaling limit appears to differ qualitatively from the results of Sec. 3. The numerical data indicate that the dependence of the critical exponents on the parameters of the defect is not a continuous one. A conjecture for the operator content based on finite-size calculations for chains with a length of up to sixteen sites is presented. It can be expressed in terms of irreps of the VA with central charge $c=\frac{1}{2}$ involving only one primary field in each case.

The main results of this paper are summarized in the concluding Sec. 5. On top of this some questions that remained open are discussed in this place. The two Appendices consist of proofs concerning parts of the exact solution in Sec. 3 that had been omitted therein for the sake of the reader.

\section{2 'Ordinary' and 'generalized' defects}

In terms of the Pauli matrices $\sigma^{x}, \sigma^{y}$, and $\sigma^{z}$, the $N$-site ferromagnetic quantum Ising Hamiltonian reads

$$
H=-\frac{1}{2} \sum_{j=1}^{N}\left[\sigma_{j}^{z}+\lambda \sigma_{j}^{x} \sigma_{j+1}^{x}\right]
$$

where $\lambda$ plays the role of the inverse temperature and the meaning of $\sigma_{N+1}^{x}$ has to be fixed by an appropriate boundary condition (BC). In this article only those BCs (or 'defects') will be considered that can be expressed in this way and where in addition $\sigma_{N+1}^{x}$ depends only on variables that live on the first site. Thus in Eq. (2.1) only (at most) two adjacent sites are coupled directly. The Hamiltonian (2.1) with a proper choice of the boundary term enjoys a global $\mathbf{Z}_{2}$-invariance. It commutes with the 'charge' operator $\hat{Q}$ defined by

$$
\hat{Q}=\prod_{j=1}^{N} \sigma_{j}^{z} \quad, \quad \hat{Q}^{2}=\mathbf{1},
$$

where 1 denotes the identity operator. One can therefore divide the spectrum of $H$ (2.1) into two sectors corresponding to an eigenvalue $(-1)^{q}$ of $\hat{Q}$ with $q \in\{0,1\}$. The system described by $H$ (2.1) is the Hamiltonian limit of the classical two-dimensional Ising model that exhibits a continuous phase transition at $\lambda=1$ and the finite-size scaling limit of the spectrum is known for various different BCs, such as toroidal, free, fixed and mixed BCs [16, 8, 17] and for the case of an arbitrary number of isolated ('ordinary') defects [1] 12. Before starting with the subject of this paper and defining new possible choices of BCs, let us have a look to the formerly used BCs and the results that have been obtained for these.

One already mentioned group of BCs is the so-called 'one defect' [10] (in the sequel the notion 'ordinary' defect will be used to avoid confusion with the generalized defects) of strength $e_{x}$, where 
one defines $\sigma_{N+1}^{x}$ to be (cf. Eq.(1.2) $)$

$$
\sigma_{N+1}^{x}=e_{x} \cdot \sigma_{1}^{x}
$$

with $e_{x} \in \mathbf{R}$, which in the two-dimensional model corresponds to a change of the coupling constant on a half-infinite line [10. For special values of the parameter $e_{x}$, this includes most of the previously examined possible boundary terms. There are two distinguished choices for $\sigma_{N+1}^{x}$ in (2.1) that correspond to periodic and antiperiodic BCs given by $e_{x}=1$ and $e_{x}=-1$, respectively. These are the sole BCs that allow the definition of a translation operator that commutes with $H$ (2.1) and therefore are called toroidal BCs (see [18). Free BCs occur in this context as the case $e_{x}=0$. The also previously examined so-called fixed [16] BCs - which by performing a duality transformation can be shown to yield the same results as free BCs - as well as mixed BCs [16. cannot be written in this simple form, they are obtained by attaching constant terms to the two ends, respectively to one end (leaving the other end free), of the chain.

All the above-mentioned BCs (or defects) have in common that the Hamiltonian $H$ (2.1) commutes with the charge operator $\hat{Q}(2.2)$ and beyond it can be written as a bilinear expression in fermionic operators. The last property ensures that the system stays finite-size integrable in each case. Therefore, the finite-size scaling limit spectra are known exactly for all these cases. In general, they can be expressed in terms of irreps of a 'shifted' $u(1)$ KMA [14, 19] that contains a VA with central extension $c=1$. For the special cases of free (as also for fixed and mixed) BCs it is also possible to use one VA with central charge $c=\frac{1}{2}$ to describe the spectrum in the finitesize scaling limit ( 8,17 , see also Sec. 4), whereas for toroidal boundary conditions one has two commuting VAs with $c=\frac{1}{2}[8]$ due to translational invariance.

To be more precise, let me reformulate the contents of the last paragraph in a more accurate way. As already mentioned, the operator content for free and toroidal BCs can be expressed [8, 17. in terms of unitary irreps of one respective two commuting VAs with $c=\frac{1}{2}$. For general central extension $c$, the Virasoro generators $L_{m}, m \in \mathbf{Z}$, fulfill the commutation relations

$$
\left[L_{m}, L_{n}\right]=(m-n) L_{m+n}+\frac{c}{12} m\left(m^{2}-1\right) \delta_{m+n, 0}
$$

with $m, n \in \mathbf{Z}$. The VA with central charge $c=\frac{1}{2}$ has only three unitary irreps [7] corresponding to a highest weight $\Delta \in\left\{0, \frac{1}{16}, \frac{1}{2}\right\}$. The character functions $\chi_{\Delta}(z)=\operatorname{tr}\left(z^{L_{0}}\right)$ in this case are given by [20, 21]

$$
\begin{aligned}
& \chi_{0}(z)=\sum_{n \in \mathbf{Z}}\left(z^{\left(12 n^{2}+n\right)}-z^{\left(12 n^{2}+7 n+1\right)}\right) \cdot \Pi_{V}(z)=\sum_{n \in \mathbf{Z}} z^{4 n^{2}+n} \cdot \Pi_{V}\left(z^{2}\right) \\
& \chi_{\frac{1}{16}}(z)=z^{\frac{1}{16}} \cdot \sum_{n \in \mathbf{Z}}\left(z^{\left(12 n^{2}+2 n\right)}-z^{\left(12 n^{2}+14 n+4\right)}\right) \cdot \Pi_{V}(z)=z^{\frac{1}{16}} \cdot \prod_{m=1}^{\infty}\left(1-z^{2 m}\right) \cdot \Pi_{V}(z) \\
& \chi_{\frac{1}{2}}(z)=z^{\frac{1}{2}} \cdot \sum_{n \in \mathbf{Z}}\left(z^{\left(12 n^{2}+5 n\right)}-z^{\left(12 n^{2}+11 n+2\right)}\right) \cdot \Pi_{V}(z)=z^{\frac{1}{2}} \cdot \sum_{n \in \mathbf{Z}} z^{4 n^{2}+3 n} \cdot \Pi_{V}\left(z^{2}\right) .
\end{aligned}
$$

Here, $\Pi_{V}(z)$ denotes the function generating the number of partitions, which is given as an infinite product by the expression

$$
\Pi_{V}(z)=\prod_{m=1}^{\infty}\left(\frac{1}{1-z^{m}}\right)
$$

The partition functions $\mathcal{T}_{q}^{ \pm}(z, \bar{z})$ resp. $\mathcal{F}_{q}(z)$ for the finite-size scaling spectra of the Hamiltonian $H$ (2.1) in the charge sector $q, q \in\{0,1\}$ are defined by [8]

$$
\begin{aligned}
& \mathcal{T}_{q}^{ \pm}(z, \bar{z})=\lim _{N \rightarrow \infty} \sum_{k, P} z^{\frac{1}{2}\left(\bar{T}_{q ; k}^{ \pm}(P, N)+P\right)} \bar{z}^{\frac{1}{2}\left(\bar{T}_{q ; k}^{ \pm}(P, N)-P\right)} \\
& \bar{T}_{q ; k}^{ \pm}(P, N)=\frac{N}{2 \pi}\left(E_{q ; k}^{ \pm}(P, N)-E_{0 ; 0}^{+}(0, N)\right)
\end{aligned}
$$


for toroidal BCs and [8, 17]

$$
\begin{aligned}
& \mathcal{F}_{q}(z)=\lim _{N \rightarrow \infty} \sum_{k} z^{\bar{F}_{q ; k}(N)} \\
& \bar{F}_{q ; k}(N)=\frac{N}{\pi}\left(E_{q ; k}^{f}(N)-E_{0 ; 0}^{f}(N)\right)
\end{aligned}
$$

for free BCs. Here, $E_{q ; k}^{ \pm}(P, N)$ denotes the $k^{t h}$ eigenvalue of the Hamiltonian (2.1) with periodic $(+)$ - resp. antiperiodic $(-)$ - BCs in the charge sector $q$ and with the momentum (eigenvalue of the translation operator) $P$ and the sum covers the whole spectrum of $H$ (2.1). The analogue is true for the energies $E_{q: k}^{f}(N)$ for free BCs, except that in this case there is no translation operator commuting with $H$ (2.1). The partition functions (2.7) and (2.8) are then given in terms of the character functions tabulated in Eq. (2.5) by 8

$$
\begin{aligned}
& \mathcal{T}_{0}^{+}(z, \bar{z})=\chi_{0}(z) \chi_{0}(\bar{z})+\chi_{\frac{1}{2}}(z) \chi_{\frac{1}{2}}(\bar{z}) \\
& \mathcal{T}_{1}^{-}(z, \bar{z})=\chi_{\frac{1}{2}}(z) \chi_{0}(\bar{z})+\chi_{0}(z) \chi_{\frac{1}{2}}(\bar{z}) \\
& \mathcal{T}_{0}^{-}(z, \bar{z})=\mathcal{T}_{1}^{+}(z, \bar{z})=\chi_{\frac{1}{16}}(z) \chi_{\frac{1}{16}}(\bar{z})
\end{aligned}
$$

and by 8,17

$$
\mathcal{F}_{0}(z)=\chi_{0}(z) \quad, \quad \mathcal{F}_{1}(z)=\chi_{\frac{1}{2}}(z)
$$

For completeness, let me state the results for fixed and mixed BCs. The Hamiltonians for these BCs can be summarized in the form

$$
H\left(f_{1}, f_{N}\right)=-\frac{1}{2}\left(\sum_{j=1}^{N-1}\left[\sigma_{j}^{x} \sigma_{j+1}^{x}+\sigma_{j}^{z}\right]+\sigma_{N}^{z}+f_{1} \cdot \sigma_{1}^{x}+f_{N} \cdot \sigma_{N}^{x}\right)
$$

with $f_{1}, f_{N} \in\{1,0,-1\}$. Here, the different sectors of fixed BCs correspond to the choices $f_{1}= \pm 1$ and $f_{N}= \pm 1$, whereas mixed BCs are defined by $f_{1}=0$ and $f_{N}= \pm 1$ (or, equivalently $f_{N}=0$ and $f_{1}= \pm 1$ ). The partition functions for the finite-size scaling spectra in each case are defined relative to the ground-state of the chain with free BCs as in Eq. (2.8). For fixed BCs [8], they are given by $\chi_{0}(z)$ for equal fixed boundary spins (that is for $H(1,1)$ and $H(-1,-1)$ ) and by $\chi_{\frac{1}{2}}(z)$ for different fixed boundary spins (that is for $H(1,-1)$ and $H(1,-1)$ ) at the two ends of the chain, with these two BCs being related to the two charge sectors of free BCs by means of a duality transformation. In the case of mixed $\mathrm{BCs}[\underline{8}]$, one obtains $\chi_{\frac{1}{16}}(z)$ for the properly defined (that is, a non-universal surface term has to be taken into account) partition function of the finite-size scaling spectrum of $H(0, \pm 1)$ (2.11).

Now, let me come back to the general case of one 'ordinary' defect of strength $e_{x}(2.3)$. Instead of the Hamiltonian $H\left(e_{x}\right)$ (2.1) with the defect $e_{x}$ (2.3) one considers the mixed-sector Hamiltonian $\tilde{H}\left(e_{x}\right)[12$

$$
\tilde{H}(\kappa)=H\left(e_{x}\right) \cdot P_{0}+H\left(-e_{x}\right) \cdot P_{1},
$$

where $P_{q}$ denotes the projection operator

$$
\left.P_{q}=\frac{1}{2} \cdot\left(\hat{Q}+(-1)^{q}\right)\right)
$$

onto the eigenspace belonging to an eigenvalue $(-1)^{q}$ of the charge operator $\hat{Q}(2.2), q \in\{0,1\}$. In this way one avoids the appearance of a non-local number operator [12] in the Hamiltonian. Of course, one can reconstruct the spectrum of $H$ (2.1) from the knowledge of the spectrum of $\tilde{H}$ (2.12), thus no information has been lost. The partition function $\mathcal{E}\left(e_{x}, z\right)$ of the finite-size scaling 
spectra (relative to the ground-state energy for periodic BCs $\left(e_{x}=1\right)$, cf. [12]) for the chain with one defect of strength $e_{x}$ is then defined by (cf. Eqs. (2.7) and (2.8))

$$
\begin{aligned}
\mathcal{E}\left(e_{x}, z\right) & =\lim _{N \rightarrow \infty} \sum_{k} z^{\bar{E}_{k}\left(e_{x}, N\right)} \\
\bar{E}_{k}\left(e_{x}, N\right) & =\frac{N}{2 \pi} \cdot\left(E_{k}\left(e_{x}, N\right)+A_{1}\left(e_{x}\right)-E_{0}(1, N)\right),
\end{aligned}
$$

where $E_{k}\left(e_{x}, N\right)$ denotes the $k^{t h}$ eigenvalue of the $N$-site Hamiltonian $\tilde{H}$ (2.12) with the defect $e_{x}$ (2.3). The non-universal surface-energy term $A_{1}\left(e_{x}\right)$ that occurs in the expansion of the lowest eigenvalue $E_{0}\left(e_{x}\right)$ of the Hamiltonian (2.12) with the defect $e_{x}$ 22, 23.

$$
-E_{0}\left(e_{x}\right)=A_{0} \cdot N+A_{1}\left(e_{x}\right)+A_{2}\left(e_{x}\right) \cdot N^{-1}+o\left(N^{-1}\right)
$$

has been subtracted (the bulk term $A_{0}=\frac{2}{\pi}$ of course is not affected by a defect that alters only one term in the Hamiltonian (2.12) $)$. The dependence of this surface term on $e_{x}$ is known analytically 12 and it is given by

$$
A_{1}\left(e_{x}\right)=\frac{1+e_{x}^{2}}{\pi e_{x}} \arctan \left(e_{x}\right)-\frac{1}{2},
$$

thence in particular $A_{1}(0)=\frac{1}{\pi}-\frac{1}{2}$ and $A_{1}( \pm 1)=0$, as necessary for toroidal BCs. Furthermore, the values of $A_{2}\left(e_{x}\right)$ in these cases are given by [8] $A_{2}(1)=\pi / 12, A_{2}(-1)=-\pi / 6$, and $A_{2}(0)=\pi / 48$.

The finite-size scaling limit spectra $\mathcal{E}\left(e_{x}, z\right)$ 2.14 can be expressed 12] in terms of character functions of unitary irreps of a shifted $u(1)$ KMA. For this purpose define the $u(1)$ KMA by the commutation rules

$$
\begin{aligned}
& {\left[T_{m}, T_{n}\right]=m \cdot \delta_{m+n, 0}} \\
& {\left[T_{m}, L_{n}\right]=m \cdot T_{m+n}}
\end{aligned}
$$

with $m, n \in \mathbf{Z}$, where the operators $L_{m}$ generate a VA (2.4) with the central charge $c=1$. The $L_{m}$ result from the $T_{m}$ by Sugawaras construction 24] through $L_{m}=\frac{1}{2} \sum_{r \in \mathbf{Z}}: T_{m-r} T_{r}:$, where the colons denote normal ordering. One then introduces a shift $\varphi$ by means of the automorphism

$$
\begin{aligned}
& T_{m} \longmapsto \tilde{T}_{m}(\varphi)=T_{m}+\varphi \cdot \delta_{m, 0} \\
& L_{m} \longmapsto \tilde{L}_{m}(\varphi)=L_{m}+\varphi \cdot T_{m}+\frac{1}{2} \varphi^{2} \cdot \delta_{m, 0}
\end{aligned}
$$

that leaves the commutation relations (2.17) and (2.4) invariant. The unitary highest weight irreps of this algebra have the character functions

$$
\chi_{t}(z, y)=\operatorname{tr}\left(z^{\tilde{L}_{0}(\varphi)} \cdot y^{\tilde{T}_{0}(\varphi)}\right)=z^{\frac{1}{2}(t+\varphi)^{2}} \cdot \Pi_{V}(z) \cdot y^{t+\varphi},
$$

indexed by a single real number $t \in \mathbf{R}$ which is the eigenvalue of $T_{0}$. Here, $\Pi_{V}(z)$ is the function previously defined in Eq. (2.6). In the physical application one is interested in the eigenvalue of $\tilde{L}_{0}(\varphi)$ in Eq. (2.19) only, therefore one uses the character functions setting $y=1$ and thereby leaving the actual value of $t$ (there are in general two different ones) unspecified.

The partition functions $\mathcal{E}\left(e_{x}, z\right)$ (2.14) of the finite-size scaling spectra for the Hamiltonian $\tilde{H}$ (2.12) with one defect of strength $e_{x}$ are then given by

$$
\mathcal{E}\left(e_{x}, z\right)=\operatorname{tr}\left(z^{\tilde{L}_{0}\left(\varphi\left(e_{x}\right)\right)}\right)=\sum_{n \in \mathbf{Z}} z^{\frac{1}{2}\left(n+\varphi\left(e_{x}\right)\right)^{2}} \cdot \Pi_{V}(z)
$$

with $\varphi\left(e_{x}\right)$ determined by 11$]$

$$
\varphi\left(e_{x}\right)=\frac{1}{4}-\frac{1}{\pi} \arctan \left(e_{x}\right)
$$


One recognizes that this is an infinite sum of characters of irreps of the $\varphi$ - shifted $u(1)$ KMA (2.18). Equivalently one could interpret this as a single irrep of the shifted $\frac{p}{q}=\frac{1}{2}$ higher symmetry algebra [14.

After this short résumé of known facts about the Ising quantum chain let me come back to the purpose of this article. As already defined in Eq. 1.2) of the preceding section, the notion of a 'generalized' defect means that one introduces a boundary term by defining $\sigma_{N+1}^{x}$ in Eq. (2.1) to be any expression subject to the sole requirement that it involves only variables living on the first site of the chain (and, of course, that the Hamiltonian (2.1) stays hermitian). The most universal term compatible with this condition will obviously be of the form (1.2), thus one has four real parameters that determine the defect. It is clear that all those defects with $e_{1}$ or $e_{z}$ different from zero will destroy the global $\mathbf{Z}_{2}$ symmetry of the chain. Therefore, one can distinguish between those defects that preserve the symmetry and those, which break it (for more complicated models that the Ising model, there might be different degrees of symmetry breaking by defects that one would have to consider separately). More important for the analysis is the question, if the defect will influence the finite-size integrability property of the chain, thus this will be the classification of the 'generalized' defects that will be used in what follows.

It is not the aim of this paper to exhaust all possible choices in Eq. (1.2), but rather to restrict to the examination of two generic cases, each consisting of two-parameter families of generalized defects. In the subsequent section, the most general defect preserving the global $\mathbf{Z}_{2}$ symmetry of the chain, that is $e_{1}=e_{z}=0$ in Eq. (1.2), will be studied. This always leads to an finite-size integrable Hamiltonian (however, it is not the most general type of defect doing this, another example for defects that share this property are for instance given by taking in Eq. (1.2) $e_{x}=e_{y}=e_{z}=0$ and $e_{1}$ arbitrary, which for $e_{1} \neq 0$ break the $\mathbf{Z}_{2}$ symmetry). A completely different type of defect is considered in Sec. 4. This is given by letting in Eq. (1.2) $e_{1}$ and $e_{y}$ be equal to zero and the other two coefficients unspecified. The defects of this type have the property to destroy both the global symmetry and the finite-size integrability of the chain as long as $e_{z}$ does not vanish.

\section{The exact solution for one finite-size integrable general- ized defect}

Consider the two-parameter family of Hamiltonians

$H(\alpha, \phi)=-\frac{1}{2}\left(\sum_{j=1}^{N-1}\left[\sigma_{j}^{z}+\left(\sigma_{j}^{+}+\sigma_{j}^{-}\right) \cdot\left(\sigma_{j+1}^{+}+\sigma_{j+1}^{-}\right)\right]+\sigma_{N}^{z}+\alpha \cdot\left(\sigma_{N}^{+}+\sigma_{N}^{-}\right) \cdot\left(e^{i \phi} \sigma_{1}^{+}+e^{-i \phi} \sigma_{1}^{-}\right)\right)$,

where $N$ denotes the number of sites, $\sigma_{j}^{ \pm}=\frac{1}{2}\left(\sigma_{j}^{x} \pm i \sigma_{j}^{y}\right)$, and $\alpha \in \mathbf{R}, \phi \in[0, \pi)$. This corresponds to taking in Eq. (1.2) $e_{1}=e_{z}=0$ and $e_{x}=\alpha \cos (\phi), e_{y}=-\alpha \sin (\phi)$. The Hamiltonian $H(\alpha, \phi)$ (3.1) for all values of the parameters commutes with the charge operator $\hat{Q}$ (2.2) defined in Sec. 2 . In order to avoid the appearance of a nonlocal number operator and in complete analogy to the known case $\phi=0$ [11] (see also Sec. 2), one considers the mixed sector Hamiltonian $\tilde{H}(\alpha, \phi)$ (2.13)

$$
\tilde{H}(\alpha, \phi)=H(\alpha, \phi) \cdot P_{0}+H(-\alpha, \phi) \cdot P_{1} .
$$

Here, the $P_{q}, q \in\{0,1\}$, are the projection operators formerly defined in Eq. 2.13). After performing a Jordan-Wigner transformation [5]

$$
\begin{aligned}
c_{k} & =\left(\prod_{j=1}^{k-1} \sigma_{j}^{z}\right) \sigma_{k}^{-}=\left(\prod_{j=1}^{k-1} \exp \left(i \pi \sigma_{j}^{-} \sigma_{j}^{+}\right)\right) \sigma_{k}^{-} \\
c_{k}^{\dagger} & =\left(\prod_{j=1}^{k-1} \sigma_{j}^{z}\right) \sigma_{k}^{+}=\left(\prod_{j=1}^{k-1} \exp \left(-i \pi \sigma_{j}^{-} \sigma_{j}^{+}\right)\right) \sigma_{k}^{+}
\end{aligned}
$$


the Hamiltonian $\tilde{H}(\alpha, \phi)$ (3.2) reads

$$
\tilde{H}(\alpha, \phi)=\frac{N}{2}+\sum_{j, k=1}^{N}\left[B_{j, k}(\alpha, \phi) c_{j}^{\dagger} c_{k}+\frac{1}{2}\left(B_{j, k}^{\prime}(\alpha, \phi) c_{j}^{\dagger} c_{k}^{\dagger}+B_{k, j}^{\prime *}(\alpha, \phi) c_{j} c_{k}\right)\right],
$$

where the two $N \times N$ matrices $B(\alpha, \phi)$ and $B^{\prime}(\alpha, \phi)$ are given by

$$
\begin{aligned}
B_{j, k}(\alpha, \phi)= & -\delta_{j, k}+\frac{1}{2} \delta_{j+1, k}\left(1-\delta_{j, N}\right)+\frac{1}{2} \delta_{j, k+1}\left(1-\delta_{k, N}\right) \\
& -\frac{\alpha}{2}\left(e^{i \phi} \delta_{j, 1} \delta_{k, N}+e^{-i \phi} \delta_{j, N} \delta_{k, 1}\right) \\
B_{j, k}^{\prime}(\alpha, \phi)= & \frac{1}{2} \delta_{j+1, k}\left(1-\delta_{j, N}\right)-\frac{1}{2} \delta_{j, k+1}\left(1-\delta_{k, N}\right)+\frac{\alpha}{2} e^{i \phi}\left(\delta_{j, 1} \delta_{k, N}-\delta_{j, N} \delta_{k, 1}\right)
\end{aligned}
$$

and the fermionic operators $c_{k}$ and $c_{k}^{\dagger}$ fulfill the anticommutation relations

$$
\begin{aligned}
& \left\{c_{k}, c_{k^{\prime}}\right\}=\left\{c_{k}^{\dagger}, c_{k^{\prime}}^{\dagger}\right\}=0 \\
& \left\{c_{k}, c_{k}^{\prime \dagger}\right\}=\delta_{k, k^{\prime}} .
\end{aligned}
$$

The matrices $B(\alpha, \phi)$ and $B^{\prime}(\alpha, \phi)$ have the properties

$$
\begin{aligned}
B^{\dagger}(\alpha, \phi) & =B(\alpha, \phi) \\
B^{\prime \dagger}(\alpha, \phi) & =-B^{\prime *}(\alpha, \phi)=-B^{\prime}(\alpha,-\phi)
\end{aligned}
$$

and, in particular, are not real except for $\phi=0$ or $\alpha=0$ which correspond to the previously examined case of one (usual) defect 11].

In the sequel the Hamiltonian $\tilde{H}(\alpha, \phi)$ (3.4) which is bilinear in the fermionic operators $c_{k}$ and $c_{k}^{\dagger}$ (3.3) shall be diagonalized to the form

$$
\tilde{H}(\alpha, \phi)=\sum_{j=1}^{N} \Lambda_{j}(\alpha, \phi) a_{j}^{\dagger} a_{j}+E_{0}(\alpha, \phi, N)
$$

by means of a Bogoliubov transformation, where the operators $a_{k}$ and $a_{k}^{\dagger}$ fulfill the same anticommutation relations as $c_{k}$ and $c_{k}^{\dagger}\left(\right.$ (3.6) , respectively, and the $\Lambda_{j}(\alpha, \phi)$ are real numbers for all $j=1, \ldots, N$ due to the hermiticity of the Hamiltonian. $E_{0}(\alpha, \phi, N)$ denotes the ground-state energy that depends on the definition of the vacuum. For this purpose we make the ansatz

$$
\begin{aligned}
& a_{k}=\sum_{j=1}^{N}\left(g_{k, j} c_{j}+h_{k, j}^{*} c_{j}^{\dagger}\right) \\
& a_{k}^{\dagger}=\sum_{j=1}^{N}\left(g_{k, j}^{*} c_{j}^{\dagger}+h_{k, j} c_{j}\right)
\end{aligned}
$$

with $k=1, \ldots, N$ and (in general) complex coefficients $g_{k, j}, h_{k, j} \in \mathbf{C}$. This generalization compared to the transformation used in [5, 11] is enforced by the non-reality of the Hamiltonian (3.4). From the anticommutation relations for the operators $a_{k}$ and $a_{k}^{\dagger}(\underline{3.6})$, one yields conditions on these coefficients which are in fact equivalent to the $2 N \times 2 N$ matrix $U$ defined by

$$
U=\left(\begin{array}{cc}
g & h^{*} \\
h & g^{*}
\end{array}\right)
$$

being unitary. Here, $g$ and $h$ denote $N \times N$ matrices with the entries $g_{k, j}$ and $h_{k, j}(k, j=1, \ldots, N)$, respectively. In other words, one has to find a unitary transformation $U$ that diagonalizes the Hamiltonian (3.4) with the additional property

$$
C U C=U^{*},
$$


$C$ denoting the $2 N \times 2 N$ matrix

$$
C=\left(\begin{array}{ll}
\mathbf{0}_{N} & \mathbf{1}_{N} \\
\mathbf{1}_{N} & \mathbf{0}_{N}
\end{array}\right) \quad, \quad C^{2}=\mathbf{1}_{2 N}
$$

Note that as a consequence of this property the determinant of $U$ is restricted to the values $\operatorname{det} U \in\{1,-1\}$.

Necessary conditions for the existence of a transformation as desired are the $N$ equations

$$
\left[a_{k}, \tilde{H}(\alpha, \phi)\right]=\Lambda_{k}(\alpha, \phi) \cdot a_{k} \quad, \quad k=1, \ldots, N,
$$

which, using Eqs. (3.4) and (3.9), after some calculations result in the equations $(k=1, \ldots, N)$

$$
\begin{aligned}
& \Lambda_{k} \cdot \vec{g}_{k}=\vec{g}_{k} \cdot B-\vec{h}_{k}^{*} \cdot B^{\prime *} \\
& \Lambda_{k} \cdot \vec{h}_{k}^{*}=\vec{g}_{k} \cdot B^{\prime}-\vec{h}_{k}^{*} \cdot B^{*}
\end{aligned}
$$

together with their complex conjugated. For convenience, the dependency on the parameters $\alpha$ and $\phi$ was dropped and the row vectors $\vec{g}_{k}=\left(g_{k, 1}, \ldots, g_{k, N}\right)$ and $\vec{h}_{k}=\left(h_{k, 1}, \ldots, h_{k, N}\right)$ were used. Introducing the notations

$$
\begin{aligned}
& \vec{\Phi}_{k}=\frac{1}{\sqrt{2}}\left(g_{k, 1}+h_{k, 1}, \ldots, g_{k, N}+h_{k, N}, g_{k, 1}^{*}+h_{k, 1}^{*}, \ldots, g_{k, N}^{*}+h_{k, N}^{*}\right) \\
& \vec{\Psi}_{k}=\frac{i}{\sqrt{2}}\left(g_{k, 1}-h_{k, 1}, \ldots, g_{k, N}-h_{k, N}, h_{k, 1}^{*}-g_{k, 1}^{*}, \ldots, h_{k, N}^{*}-g_{k, N}^{*}\right)
\end{aligned}
$$

and

$$
\widehat{B}=\left(\begin{array}{cc}
B & B^{\prime} \\
-B^{\prime *} & -B^{*}
\end{array}\right)
$$

with $\widehat{B}$ being hermitian, Eqs. (3.14) become

$$
\begin{aligned}
& \Lambda_{k} \cdot \vec{\Phi}_{k}=-i \vec{\Psi}_{k} \cdot \widehat{B} \\
& \Lambda_{k} \cdot \vec{\Psi}_{k}=i \vec{\Phi}_{k} \cdot \widehat{B} .
\end{aligned}
$$

One therefore derives the following eigenvalue equations for the squares of the fermion frequencies $\Lambda_{k}(k=1, \ldots, N)$

$$
\begin{aligned}
\Lambda_{k}^{2} \cdot \vec{\Phi}_{k} & =\vec{\Phi}_{k} \cdot \widehat{A}=\vec{\Phi}_{k} \cdot \widehat{B}^{2} \\
\Lambda_{k}^{2} \cdot \vec{\Psi}_{k} & =\vec{\Psi}_{k} \cdot \widehat{A}=\vec{\Psi}_{k} \cdot \widehat{B}^{2},
\end{aligned}
$$

where $\widehat{A}=\widehat{B}^{2}$ is an — at least — positive semi-definite hermitian $2 N \times 2 N$ matrix given explicitly by

$$
\widehat{A}(\alpha, \phi)=\left(\begin{array}{cc}
A(\alpha, \phi) & A^{\prime}(\alpha, \phi) \\
A^{\prime *}(\alpha, \phi) & A^{*}(\alpha, \phi)
\end{array}\right)
$$

with the two $N \times N$ matrices $A$ and $A^{\prime}$

$$
\begin{aligned}
A_{j, k}(\alpha, \phi)= & \delta_{j, k} \cdot\left(2-\frac{1-\alpha^{2}}{2}\left(\delta_{j, 1} \delta_{k, 1}+\delta_{j, N} \delta_{k, N}\right)\right)-\delta_{j+1, k} \cdot\left(1-\delta_{j, N}\right)-\delta_{j, k+1} \cdot\left(1-\delta_{k, N}\right) \\
& +\alpha e^{i \phi} \delta_{j, 1} \delta_{k, N}+\alpha e^{-i \phi} \delta_{j, N} \delta_{k, 1}-\frac{i \alpha \sin (\phi)}{2} \cdot\left(\delta_{j, 2} \delta_{k, N}-\delta_{j, N} \delta_{k, 2}\right) \\
A_{j, k}^{\prime}(\alpha, \phi)= & \frac{\alpha^{2} e^{2 i \phi}-1}{2} \delta_{j, 1} \delta_{k, 1}-\frac{\alpha^{2}-1}{2} \delta_{j, N} \delta_{k, N}+\frac{i \alpha \sin (\phi)}{2} \cdot\left(\delta_{j, 2} \delta_{k, N}+\delta_{j, N} \delta_{k, 2}\right) .
\end{aligned}
$$

Note the following property of $\widehat{A}(\alpha, \phi)$

$$
C \widehat{A}(\alpha, \phi) C=\widehat{A}^{*}(\alpha, \phi) .
$$


The unitarity condition on $U$ (3.10) leads to the restrictions

$$
\vec{\Phi}_{j} \cdot \vec{\Phi}_{k}^{\dagger}=\vec{\Psi}_{j} \cdot \vec{\Psi}_{k}^{\dagger}=\delta_{j, k} \quad, \quad \vec{\Phi}_{j} \cdot \vec{\Psi}_{k}^{\dagger}=0,
$$

or, equivalently, to the unitarity of the matrix $\widehat{U}$,

$$
\widehat{U}=\left(\begin{array}{cc}
g+h & (g+h)^{*} \\
i(g-h) & (i(g-h))^{*}
\end{array}\right)=T \cdot U
$$

that is built from the row vectors $\vec{\Phi}_{k}$ and $\vec{\Psi}_{k}, k=1, \ldots, N$. Here, $T$ is the unitary matrix

$$
T=\frac{1}{\sqrt{2}}\left(\begin{array}{cc}
\mathbf{1}_{N} & \mathbf{1}_{N} \\
i \mathbf{1}_{N} & -i \mathbf{1}_{N}
\end{array}\right) .
$$

In addition, the fact that $U$ has to comply with Eq. (3.11) leads to the conditions

$$
\vec{\Phi}_{k} \cdot C=\vec{\Phi}_{k}^{*} \quad, \quad \vec{\Psi}_{k} \cdot C=\vec{\Psi}_{k}^{*} \quad, \quad k=1, \ldots, N,
$$

or, equivalently,

$$
\widehat{U} \cdot C=\widehat{U}^{*} .
$$

However, in Appendix A it is shown that the latter conditions can be fulfilled by a simple change of basis always. Thus it is possible to reconstruct from the knowledge of $\widehat{U}$ the transformation $U$ (3.10) by $U=T^{\dagger} \widehat{U}$ and thereby the Eqs. (3.17), (3.22), and (3.25) in fact are necessary and sufficient to determine the Bogoliubov transformation (3.9).

In this way the determination of the $\Lambda_{k}$ - up to a sign - has been transformed to the problem of computing the eigenvalues of a $2 N \times 2 N$ hermitian matrix and, furthermore, a Bogoliubov transformation (3.9) can be constructed from the knowledge of the eigenvectors. The only thing that has been missed so far is the degeneration of the spectrum of $\widehat{A}$ that has to exist if the Eqs. (3.18) can be solved consistently. This is the purpose of Appendix B, where the origin of this property - obviously a symmetry hidden in the equations - is examined. One might think that this is not necessary because it follows from the ansatz (3.9), but it is the aim to show that this ansatz can always be used and does not lead to equations that cannot be solved. The question about the sign of the $\Lambda_{k}$ can be answered as follows. A change of the sign of $\Lambda_{k}$ corresponds to an exchange of the corresponding creation and annihilation operators $a_{k}^{\dagger}$ and $a_{k}$, which is a special Bogoliubov transformation (see also Appendix B), together with a shift in the ground-state energy $E_{0}$ (3.8). In order to let the vacuum (the state annihilated by all annihilation operators) be the state with lowest energy, one chooses all $\Lambda_{k}$ to be non-negative.

Let me stop at this point for a short comment. From Eq. (3.20), one recognizes that the matrix $A^{\prime}(\alpha, \phi)$ does not vanish for $\phi=0$ as one might have expected. This is of course due to the choice of the basis (3.15) and it is easy to find a Bogoliubov transformation (3.9) that block-diagonalizes $\widehat{A}(\alpha, 0)$ (3.19) to a form with twice the same real symmetric matrix along the diagonal. In this way the problem as usually reduces to the diagonalization of a $N \times N$ matrix [11] and one just obtains the whole spectrum doubled. Principally, the block-diagonalization remains possible in the general case $\phi \neq 0$, too, but the transformation may become arbitrarily complicated. However, in Appendix B it will be argued that the doubling of the spectrum is a general feature - as it must be to fulfill Eqs. (3.18) - not limited to the case $\phi=0$.

The eigenvalue equations (3.18) are now solved by means of an ansatz. This is done the same way as in [5, 11, 13] by

$$
\begin{aligned}
& \vec{\Phi}_{p, j}=\frac{1}{\sqrt{2}} \cdot\left(r_{j} e^{\frac{i p}{N}}+s_{j} e^{-\frac{i p}{N}}\right) \\
& \vec{\Phi}_{p, j+N}=\frac{1}{\sqrt{2}} \cdot\left(\tilde{r}_{j} e^{\frac{i p}{N}}+\tilde{s}_{j} e^{-\frac{i p}{N}}\right)
\end{aligned}
$$

with $j=1, \ldots, N$, where the eigenvectors $\vec{\Phi}_{p}$ (and likewise the eigenvalues $\Lambda_{p}$ ) are from now on indexed by the variable $p$ which will (in general) not be integer-valued. One observes that all but six of the $2 N$ linear homogeneous equations summarized in

$$
\Lambda_{p}^{2} \cdot \vec{\Phi}_{p}=\vec{\Phi}_{p} \cdot \widehat{A}(\alpha, \phi)
$$


are solved by the relation

$$
\Lambda_{p}^{2}=\left(2 \sin \left(\frac{p}{2}\right)\right)^{2}
$$

The remaining six equations then determine the possible values of $p$ through the requirement that there has to exist a solution for the variables $r_{j}, \tilde{r}_{j}, s_{j}$, and $\tilde{s}_{j}$ in the ansatz (3.27). This is obviously equivalent to demand the coefficient determinant to vanish. The computation of this determinant yields a - highly non-linear - equation for the allowed values of $p$ and therewith for the eigenvalues $\Lambda_{p}$. It is known that in this way one obtains all those eigenvalues $\Lambda_{p}$ whose squares are smaller than four 5 . However, this is sufficient to determine the finite-size scaling limit, because in that case only low-energy states enter the calculation.

Of course, one is not able to solve the equation that determines $p$ for any size $N$ of the chain. One is rather interested in the thermodynamic limit $N \rightarrow \infty$. This is performed by expanding the determinant in inverse powers of $N$, where one uses [1, 12, 13.

$$
N \cdot p=\varepsilon_{p}+O\left(N^{-1}\right) .
$$

The coefficient of the first non-vanishing power in $N^{-1}$ leads to an equation for $\varepsilon_{p}$ that determines the scaled eigenvalues in the continuum limit by

$$
\lim _{N \rightarrow \infty}\left(N \cdot \Lambda_{p}\right)=\varepsilon_{p} .
$$

The above-mentioned algebraic calculations were performed on the computer. Let me only state the result. The final equation for $\varepsilon_{p}(\alpha, \phi)$ reads

$$
\left(\left(1+\kappa^{2}\right) \cdot \cos \left(\varepsilon_{p}\right)+2 \kappa\right)^{2}=0 \quad, \quad \kappa=\alpha \cos (\phi),
$$

and one recognizes that this is just the square of the equation that one obtains for one 'ordinary' defect of strength $\kappa=\alpha \cos (\phi)$ [11, 12]. Therefore the finite-size scaling limit is the same for both systems, although they generally differ on finite chains. Thus the operator content that only depends on $\kappa$ can be read off from Eqs. (2.20) and (2.21) in Sec. 2 directly.

Therefore, exactly as for an 'ordinary' defect [12, 13, the scaled (relative to the ground-state energy $E_{0}(1,0, N)$ for periodic BCs) Hamiltonian $\widehat{\tilde{H}}(\alpha, \phi)$

$$
\widehat{\tilde{H}}(\alpha, \phi)=\lim _{N \rightarrow \infty} \frac{N}{2 \pi} \cdot\left(\tilde{H}(\alpha, \phi)-E_{0}(1,0, N)+A_{1}(\alpha, \phi)\right)
$$

is given by a generator $\tilde{L}_{0}(\varphi(\alpha \cos (\phi)))$ of a shifted $u(1)$ KMA 2.18). From the positive solutions of Eq. (3.32) one obtains the scaled values for the fermion frequencies (3.31) which have to be inserted into Eq. (3.8). This results in

$$
\begin{aligned}
\widehat{\tilde{H}}(\alpha, \phi) & =\widehat{\tilde{H}}(\kappa) \\
& =\sum_{k \in \mathbf{N}_{0}}\left(k+\frac{1}{2}-\varphi(\kappa)\right) a_{k}^{\dagger} a_{k}+\left(k+\frac{1}{2}+\varphi(\kappa)\right) b_{k}^{\dagger} b_{k}+\frac{1-2 \tilde{c}(\kappa)}{24} \\
& =\tilde{L}_{0}(\varphi(\kappa))
\end{aligned}
$$

where the shift $\varphi(\kappa)$ has the same form as for an 'ordinary' defect [11, 12] (cf. Eq. (2.21))

$$
\varphi(\kappa)=\frac{1}{4}-\frac{1}{\pi} \arctan (\kappa),
$$

with $\kappa=\alpha \cos (\phi)$. Here, Eq. 2.15) with $A_{2}(1)=\pi c / 6$ and the expansion of the ground-state energy $E_{0}(\alpha, \phi, N)$ (3.8) of the Hamiltonian $\tilde{H}(\alpha, \phi)$ (3.4)

$$
-E_{0}(\alpha, \phi, N)=A_{0} \cdot N+A_{1}(\alpha, \phi)+\frac{\pi \tilde{c}(\alpha, \phi)}{6} \cdot N^{-1}+o\left(N^{-1}\right)
$$


were used. The 'effective' central charge $\tilde{c}(\alpha, \phi)$ that also depends on $\kappa=\alpha \cos (\phi)$ only, is determined by the shift $\varphi$ (3.35) through the relation

$$
\frac{(\varphi(\kappa))^{2}}{2}=\frac{1-2 \tilde{c}(\kappa)}{24},
$$

and the fermionic operators $a_{k}$ and $b_{k}$ in Eq. (3.34) descend from the $a_{k}$ in Eq. (3.8) by means of a suitable re-numeration of the indices.

The only thing involved in the resulting expressions that does not depend on $\kappa=\alpha \cos (\phi)$ alone is the (non-universal) surface energy $A_{1}(\alpha, \phi)$. Since the ground-state energy does not obtain from the analysis performed, no analytic expression is known for this term. However, numerical investigations show that it differs from the $A_{1}(\alpha \cos (\phi))$ of Eq. (2.16) by an amount of $\frac{1}{\pi}$ at most (for $\phi=\frac{\pi}{2}$ and $\alpha \rightarrow \infty$ ), thus it resembles the function for an 'ordinary' defect. In analogy to the case of 'ordinary' defects, a generalization of this investigation to an arbitrary number of isolated generalized defects of this form at commensurate distances [11, 12] or to a generalized extended defect [15] obviously can be performed the same way as for 'ordinary' defects.

\section{Finite-size non-integrable generalized defects}

In this section another two-parameter family of Hamiltonians $\mathcal{H}(\beta, \psi)$ defined by

$$
\mathcal{H}(\beta, \psi)=-\frac{1}{2}\left(\sum_{j=1}^{N-1}\left[\sigma_{j}^{z}+\sigma_{j}^{x} \sigma_{j+1}^{x}\right]+\sigma_{N}^{z}+\beta \sigma_{N}^{x} \cdot\left(\cos (\psi) \sigma_{1}^{x}-\sin (\psi) \sigma_{1}^{z}\right)\right),
$$

will be considered. Here, $\beta \in \mathbf{R}-\{0\}$ and $\psi \in(0, \pi)$, whereby one guarantees that $\beta \sin (\psi)$ is different from zero because $\beta \sin (\psi)=0$ recovers finite-size integrable defects examined in the preceding section. As has been discussed in Sec. 2, the Hamiltonian (4.1) is not finite-size integrable under this circumstances. This is caused by the occurrence of the term $\sigma_{N}^{x} \sigma_{1}^{z}$ that not only breaks the global $\mathbf{Z}_{2}$ invariance of the Ising chain Hamiltonian (see Eq. (2.2)), but also leads to the appearance of a three-fermion coupling if a Jordan-Wigner transformation as in Eq. (3.3) is performed on $\mathcal{H}$ 4.1.

Since for this reason the methods used in the previous section can not be applied, the Hamiltonian (4.1) has been investigated by numerical calculations only. This was done by diagonalizing $\mathcal{H}$ for up to sixteen sites using an algorithm by Lanczos [25]. The scaled energy gaps $\bar{F}_{k}(\beta, \psi, N)$, defined in this case relative to free $\mathrm{BCs}$

$$
\bar{F}_{k}(\beta, \psi, N)=\frac{N}{\pi}\left(E_{k}(\beta, \psi, N)+A_{1}(\beta, \psi)-E_{0}(0,0, N)-A_{1}(0,0)\right)
$$

with $A_{1}(0,0)=\frac{1}{\pi}-\frac{1}{2}$ (2.16), have to be extrapolated to an infinite system

$$
\overline{\mathcal{F}}_{k}(\beta, \psi)=\lim _{N \rightarrow \infty} \bar{F}_{k}(\beta, \psi, N) .
$$

Here, $A_{1}(\beta, \psi)$ denotes the surface term that occurs in the expansion of the lowest eigenvalue $E_{0}(\beta, \psi, N)$ (cf. Eq. (2.15) ) of the Hamiltonian (4.1)

$$
-E_{0}(\beta, \psi, N)=A_{0} \cdot N+A_{1}(\beta, \psi)+\frac{\pi \tilde{c}(\beta, \psi)}{24} \cdot N^{-1}+o\left(N^{-1}\right),
$$

and $E_{k}(\beta, \psi, N)$ is the $k^{t h}$ eigenvalue of the $N$-site Hamiltonian $\mathcal{H}(\beta, \psi)$ (4.1). The partition function $\mathcal{F}(\beta, \psi, z)$ for the finite-size scaling spectrum is defined by

$$
\mathcal{F}(\beta, \psi, z)=\lim _{N \rightarrow \infty} \sum_{k} z^{\bar{F}_{k}(\beta, \psi, N)}
$$


where the sum extends over the whole spectrum of $\mathcal{H}$ (4.1). The use of $E_{0}(0,0, N)$ as the reference energy in Eq. (4.2) will be justified by the final expression for the partition function $\mathcal{F}(\beta, \psi, z)$ (4.5) conjectured on the basis of numerical results.

There is one disadvantage in the use of Eqs. (4.2) and (4.3) for the actual numerical computations, namely that the analytic form of the surface energy $A_{1}(\beta, \psi)$ (4.4) is not known. Therefore, one alternatively extrapolates the scaled energy gaps referred to the relative ground-state $E_{0}(\beta, \psi, N)$ and besides that determines the lowest scaled energy gap $\overline{\mathcal{F}}_{0}(\beta, \psi)$

$$
\overline{\mathcal{F}}_{0}(\beta, \psi)=\lim _{N \rightarrow \infty} \frac{N}{\pi} \cdot\left(E_{0}(\beta, \psi, N)-E_{0}(0,0, N)+A_{1}(\beta, \psi)-A_{1}(0,0)\right)
$$

from the finite-size corrections of the relative ground-state energy $E_{0}(\beta, \psi, N)$ (4.4). Knowing the 'effective central charge' $\tilde{c}(\beta, \psi)$ (4.4) is equivalent to the knowledge of $\overline{\mathcal{F}}_{0}(\beta, \psi)$, because by Eqs. (2.15) (with $A_{2}(0)=\frac{\pi c}{24}$ ) and (4.6) the relation $\left(c=\frac{1}{2}\right)$

$$
\begin{aligned}
\overline{\mathcal{F}}_{0}(\beta, \psi) & =\frac{c-\tilde{c}(\beta, \psi)}{24} \\
& =\frac{1-2 \tilde{c}(\beta, \psi)}{48}
\end{aligned}
$$

holds. This was used to numerically compute $\overline{\mathcal{F}}_{0}(\beta, \psi)$ for various choices of the parameters. For this purpose the known value of $A_{0}=\frac{2}{\pi}$ was inserted into Eq. (4.4) and the unknown surface term $A_{1}(\beta, \psi)$ (4.4) was eliminated by combining the equation for two subsequent values of $N$. The value of $\overline{\mathcal{F}}_{0}(\beta, \psi)$ then could be determined approximatively by extrapolating to infinite size $N \rightarrow \infty$, wherefore the algorithm developed by Bulirsch and Stoer ([26], see also [27]) was employed.

The numerical results are in reasonable agreement with the following conjecture for the operator content expressed through the character functions [20] $\chi_{\Delta}(z)$ (2.5) of the unitary irreps of the $c=\frac{1}{2}$ VA (2.4) corresponding to a highest weight $\Delta, \Delta \in\left\{0, \frac{1}{16}, \frac{1}{2}\right\}$. For $\beta>0$ one has

$$
\mathcal{F}(\beta, \psi, z)= \begin{cases}\chi_{0}(z) & \text { if } \psi \in\left(0, \frac{\pi}{2}\right) \\ \chi_{\frac{1}{16}}(z) & \text { if } \psi=\frac{\pi}{2} \\ \chi_{\frac{1}{2}}(z) & \text { if } \psi \in\left(\frac{\pi}{2}, \pi\right)\end{cases}
$$

wherefrom the case $\beta<0$ can be deduced using the relation

$$
\mathcal{F}(\beta, \psi, z)=\mathcal{F}(-\beta, \pi-\psi, z) .
$$

This equality follows directly from the fact that for arbitrary $N$ the Hamiltonian (4.1) possesses the property

$$
\hat{Q} \cdot \mathcal{H}(\beta, \psi) \cdot \hat{Q}=\mathcal{H}(-\beta, \pi-\psi)
$$

implying the equality of the finite-size spectra of $\mathcal{H}(\beta, \psi)$ and $\mathcal{H}(-\beta, \pi-\psi)$.

In Table 1 the obtained estimates of the lowest scaled energy gap $\overline{\mathcal{F}}_{0}(\beta, \psi)$ (4.7) together with their - rather subjective - errors for $\beta=1$ and different values of $\psi$ are presented. Table 2 contains the same thing for three fixed values of $\psi$, namely $\pi / 5, \pi / 2$, and $4 \pi / 5$, and varying $\beta$. Of course, only a small fraction of the collected data could be displayed therein. In both tables, the values deduced from the conjecture (4.8) are opposed to the numerical data. A comparison shows that the agreement is good on the whole, especially if one stays far enough away from $\beta=0$ and $\psi \in\left\{0, \frac{\pi}{2}, \pi\right\}$. Near those values of the parameters the extrapolation is difficult (and the resulting 'errors' thereby big), what can be understood from the assertion that the dependence on the parameters ceases to be continuous there. Obviously one is not able to see such an effect in a finite sample, thus the observed behaviour agrees with what one would expect. Anyhow one might have awaited possible discontinuities at those points where the symmetry of the Hamiltonian (4.1) changes, these are all the critical values mentioned above with the exception of $\psi=\frac{\pi}{2}$. In this case no obvious change of the symmetry occurs, but there might be a 'hidden' symmetry that one cannot find by inspection. In Table 3 and Table 4, numerical results for the surface energy 
Table 1: Opposition of numerical estimates and by Eq. 4.8) conjectured values of the lowest scaled energy gap $\overline{\mathcal{F}}_{0}(\beta, \psi)$ (4.6) for $\beta=1$ and different choices of the parameter $\psi$.

\begin{tabular}{|c|c|c|}
\hline$\psi$ & numerical estimate & conjectured value \\
\hline $0.1 \pi$ & $0.0005(3)$ & 0.000000 \\
$0.2 \pi$ & $0.0001(2)$ & 0.000000 \\
$0.3 \pi$ & $0.0002(3)$ & 0.000000 \\
$0.4 \pi$ & $0.001(2)$ & 0.000000 \\
$0.5 \pi$ & $0.06250(3)$ & 0.062500 \\
$0.6 \pi$ & $0.6(1)$ & 0.500000 \\
$0.7 \pi$ & $0.498(1)$ & 0.500000 \\
$0.8 \pi$ & $0.4995(3)$ & 0.500000 \\
$0.9 \pi$ & $0.5(1)$ & 0.500000 \\
\hline
\end{tabular}

Table 2: The same as in Table 1, but for three fixed values of the parameter $\psi$ and varying $\beta$.

\begin{tabular}{||r|c|c|c||}
\hline \hline \multicolumn{1}{|c|}{$\beta$} & $\psi$ & numerical estimate & conjectured value \\
\hline \hline 0.5 & $0.2 \pi$ & $0.0002(3)$ & 0.000000 \\
2.0 & $0.2 \pi$ & $0.00003(2)$ & 0.000000 \\
5.0 & $0.2 \pi$ & $0.00001(2)$ & 0.000000 \\
20.0 & $0.2 \pi$ & $0.00000(1)$ & 0.000000 \\
100.0 & $0.2 \pi$ & $0.00000(1)$ & 0.000000 \\
\hline 0.5 & $0.5 \pi$ & $0.06250(1)$ & 0.062500 \\
2.0 & $0.5 \pi$ & $0.06251(2)$ & 0.062500 \\
5.0 & $0.5 \pi$ & $0.06250(1)$ & 0.062500 \\
20.0 & $0.5 \pi$ & $0.06250(1)$ & 0.062500 \\
100.0 & $0.5 \pi$ & $0.0625(1)$ & 0.062500 \\
\hline 0.5 & $0.8 \pi$ & $0.5(1)$ & 0.500000 \\
2.0 & $0.8 \pi$ & $0.5005(3)$ & 0.500000 \\
5.0 & $0.8 \pi$ & $0.50001(2)$ & 0.500000 \\
20.0 & $0.8 \pi$ & $0.50000(1)$ & 0.500000 \\
100.0 & $0.8 \pi$ & $0.50003(2)$ & 0.500000 \\
\hline \hline
\end{tabular}

$A_{1}(\beta, \psi)$ (4.4) for various values of the parameters $\beta$ and $\psi$ are given. In this case no analytic expression is known and thus there is nothing to compare with.

Another support for the conjecture (4.8) results from the counting of the degeneracies of the excited levels ('conformal towers'). These could in all cases be seen up to the forth or fifth excitation of the lowest level. First, there are no indications of the existence of a second primary field in the operator content, that is up to the (at least) forth excited level of the relative ground-state nothing but the expected levels - the conformal tower of the ground-state - has been detected. On the other hand, the degeneracies of the conformal towers for the three possible primaries at $c=\frac{1}{2}$ differ (at the latest) in the third excitation, therefore the observed degeneracies that complied with Eq. (4.8) in all examined cases, strongly support the conjecture.

\section{Conclusions}

It was the aim of this article to examine the finite-size scaling properties of the quantum Ising chain with one 'generalized' defect (1.2), which has been introduced by an arbitrary transformation in the algebra of observables in the coupling term at one particular site of the chain. The finitesize scaling spectrum for the Ising chain equipped with the most universal defect that maintains its global $\mathbf{Z}_{2}$ symmetry was obtained analytically and proven to be the same as in the case of 
Table 3: Numerical estimates of the surface energy $A_{1}(\beta, \psi)$ (4.4) for $\beta=1$ and different values of $\psi$.

\begin{tabular}{|c|c|}
\hline$\psi$ & numerical estimate \\
\hline $0.1 \pi$ & $0.04377(2)$ \\
$0.2 \pi$ & $0.07821(1)$ \\
$0.3 \pi$ & $0.09682(3)$ \\
$0.4 \pi$ & $0.10143(3)$ \\
$0.5 \pi$ & $0.097454(3)$ \\
$0.6 \pi$ & $0.101(1)$ \\
$0.7 \pi$ & $0.095(2)$ \\
$0.8 \pi$ & $0.080(1)$ \\
$0.9 \pi$ & $0.0434(1)$ \\
\hline
\end{tabular}

Table 4: The same as in Table 3, but for three values of the parameter $\psi$ and varying $\beta$.

\begin{tabular}{||r|c|c||}
\hline \hline \multicolumn{1}{|r|}{$\beta$} & $\psi$ & numerical estimate \\
\hline \hline 0.5 & $0.2 \pi$ & $-0.09663(3)$ \\
2.0 & $0.2 \pi$ & $0.5105577(2)$ \\
5.0 & $0.2 \pi$ & $1.9502580(1)$ \\
20.0 & $0.2 \pi$ & $9.4111164(1)$ \\
100.0 & $0.2 \pi$ & $49.3993176(2)$ \\
\hline 0.5 & $0.5 \pi$ & $-0.0798648(2)$ \\
2.0 & $0.5 \pi$ & $0.522629(3)$ \\
5.0 & $0.5 \pi$ & $1.9447786(1)$ \\
20.0 & $0.5 \pi$ & $9.387026(2)$ \\
100.0 & $0.5 \pi$ & $49.368324(1)$ \\
\hline 0.5 & $0.8 \pi$ & $-0.0958(3)$ \\
2.0 & $0.8 \pi$ & $0.5105(3)$ \\
5.0 & $0.8 \pi$ & $1.95026(1)$ \\
20.0 & $0.8 \pi$ & $9.4111165(3)$ \\
100.0 & $0.8 \pi$ & $49.399318(1)$ \\
\hline \hline
\end{tabular}

one suitably chosen 'ordinary' defect and thus could be expressed in terms of irreps of a shifted $u(1)$ Kac-Moody algebra. The result is (see Eqs. (3.32)-(3.35) that for those generalized defects that do not break the global $\mathbf{Z}_{2}$ symmetry of the quantum Ising chain the partition function in the continuum limit is in fact identical to that for one ordinary defect. The generalization to an arbitrary number of defects of this form at commensurate distances obviously is completely analogous to the case of 'ordinary' defects [11, 12. Recently, a similar observation [28 has been made concerning the Ising quantum chain with three spin couplings 28] and the Ashkin-Teller model equipped with special defects [29] that break the symmetries in a similar way in both systems. This suggests that there is some sort of 'universality' for quantum chains with defects. This means that the partition function of the system will not depend on the detailed form of the defect, but only on the symmetries of the chain and the symmetry breaking caused by the defect.

In the remainder of this article another two-parameter family of generalized defects given by $\sigma_{N+1}^{x}=\beta \cdot\left(\cos (\psi) \sigma_{1}^{x}-\sin (\psi) \sigma_{1}^{z}\right)$ with $\beta \in \mathbf{R}-\{0\}$ and $\psi \in(0, \pi)$, that destroy the finite-size integrability property of the quantum Ising chain has been studied by means of numerical finite-size calculations for chains with up to sixteen sites. They suggest a qualitatively different behaviour of the finite-size scaling spectrum, which appears to depend non-continuously on the parameter $\psi$ of the defect. In this case a conjecture for the operator content involving only one primary field of a $c=\frac{1}{2}$ VA for each choice of the parameters was given and compared to the results obtained from the numerical diagonalization of the chain. For some examples of the parameters, this comparison 
is presented in the four tables, where in addition numerical estimates for the non-universal surface energy (cf. Eq. (4.4) ) are given. For $\psi=\pi / 2$, the resulting finite-size scaling spectrum is the same as for mixed BCs, whereas for $\psi \neq \pi / 2$ one obtains the finite-size scaling spectrum of one of the two sectors of fixed BCs depending on the sign of $\beta \cos (\psi)$ only. Therefore, the highest weights $\Delta$ of the $c=\frac{1}{2}$ Virasoro irreps are observed to be $\Delta=0$ for $\beta \cos (\psi)$ (strictly) positive, $\Delta=1 / 16$ in the case $\cos (\psi)=0$, and finally $\Delta=1 / 2$ for negative values of $\beta \cos (\psi)$.

In this article, not all possible generalized defects were investigated. In principle, one has four real parameters $e_{1}, e_{x}, e y$, and $e_{z}$ corresponding to a defect of the form (1.2) for the quantum Ising Hamiltonian (2.1), so the two types of defects studied in Sec. 3 and Sec. 4 correspond to $e_{1}=e_{z}=0$ and arbitrary $e_{x}$ and $e_{y}$ resp. $e_{1}=e_{y}=0$ and arbitrary values of $e_{x}$ and $e_{z}$. Thus the question, if the finite-size scaling spectra for the chain with a localized defect different from those considered herein will show the same behaviour as observed or not, remains unanswered. One might guess that this is true, since a localized defect should not affect the conformal properties except that the two $c=\frac{1}{2}$ VAs describing the translational invariant case become coupled and thereby only their direct sum appears in the spectrum generating algebra. From this one would expect that for every choice of a localized defect the spectrum generating algebra is at least a (shifted) $u(1)$ Kac-Moody algebra. Furthermore the situations of several isolated, but possibly different, generalized defects or even extended generalized defects have not been looked upon. Clearing up these points is left to future investigations.

\section{Acknowledgements}

It is a pleasure for the author to thank V. Rittenberg for substantial support as well as P. Chaselon for many fruitful discussions.

\section{Appendix A}

In this place a simple, but maybe non-obvious corollary of linear algebra shall be proven. It has been used in Sec. 3 to show that one can always find a Bogoliubov transformation (3.9) diagonalizing the Hamiltonian (3.4) to the form (3.8) and which beyond that is compatible with the conditions (3.10) and (3.11). This was necessary to guarantee that the new operators defined by Eq. (3.9) respect the fermionic algebra (3.6). For this purpose one needs only Eq. (3.21) as supposition. This enables us to state the proposition in a more general way.

Let $M$ be a hermitian $2 N \times 2 N$ matrix with the property

$$
C M C=M^{*}
$$

where $C$ denotes the matrix 3.12)

$$
C=\left(\begin{array}{ll}
\mathbf{0}_{N} & \mathbf{1}_{N} \\
\mathbf{1}_{N} & \mathbf{0}_{N}
\end{array}\right) \quad, \quad C^{2}=\mathbf{1}_{2 N}
$$

Then there exists a set $\mathcal{B}$ of $2 N$ (row) vectors $\mathcal{B}=\left\{\vec{\Theta}_{k}, k=1, \ldots, 2 N\right\}$ fulfilling the three relations

$$
\begin{aligned}
\vec{\Theta}_{j} \cdot \vec{\Theta}_{k}^{\dagger} & =\delta_{j, k} \\
\vec{\Theta}_{k} \cdot M & =\Lambda_{k} \cdot \vec{\Theta}_{k}, \Lambda_{k} \in \mathbf{R} \\
\vec{\Theta}_{k}^{*} \cdot C & =\vec{\Theta}_{k}
\end{aligned}
$$

for all $j, k \in\{1,2, \ldots, 2 N\}$.

Since $M$ is hermitian, there exists an orthonormal set $\mathcal{B}^{(0)}=\left\{\vec{\Theta}_{k}^{(0)}, k=1, \ldots, 2 N\right\}$ of eigenvectors of $M$. This means that $\mathcal{B}^{(0)}$ automatically satisfies the first two relations (A.1) and (A.2) of the proposition. The proof is based on an iterative prescription how to built from this set $\mathcal{B}^{(0)}$ 
another set $\mathcal{B}$ of orthonormal eigenvectors of $M$ that satisfies the additional third condition in the proposition.

Before commencing with that, consider an arbitrary normalized eigenvector $\vec{\Theta}$ of $M$ belonging to an eigenvalue $\Lambda$, that is

$$
\vec{\Theta} \cdot M=\Lambda \cdot \vec{\Theta} \quad, \quad \vec{\Theta} \cdot \vec{\Theta}^{\dagger}=1 \quad, \quad \Lambda \in \mathbf{R} .
$$

It follows

$$
\begin{aligned}
\left(\vec{\Theta}^{*} C\right) \cdot M & =\vec{\Theta}^{*} \cdot(C M C) \cdot C \\
& =\vec{\Theta}^{*} \cdot M^{*} \cdot C \\
& =\Lambda \cdot\left(\vec{\Theta}^{*} C\right) \\
\left(\vec{\Theta}^{*} C\right) \cdot\left(\vec{\Theta}^{*} C\right)^{\dagger} & =1
\end{aligned}
$$

that is, $\left(\vec{\Theta}^{*} C\right)$ is normalized eigenvector of $M$ to the same eigenvalue $\Lambda$.

Now, start with $\vec{\Theta}_{1}^{(0)}$. Then there are two possibilities: either the two vectors $\left(\vec{\Theta}_{1}^{(0)^{*}} \cdot C\right)$ and $\vec{\Theta}_{1}^{(0)}$ that are both eigenvectors of $M$ to the eigenvalue $\Lambda_{1}$ linear depend on each other or not. In the first case you have

$$
\vec{\Theta}_{1}^{(0) *} \cdot C=e^{2 i \gamma} \cdot \vec{\Theta}_{1}^{(0)}
$$

with $\gamma \in[0, \pi)$. The vector $\vec{\Theta}_{1}$ defined by

$$
\vec{\Theta}_{1}=e^{i \gamma} \cdot \vec{\Theta}_{1}^{(0)}
$$

has the properties

$$
\vec{\Theta}_{1}^{*} \cdot C=\vec{\Theta}_{1} \quad, \quad \vec{\Theta}_{1} \cdot \vec{\Theta}_{1}^{\dagger}=1 .
$$

Take now as the new set $\mathcal{B}^{(1)}=\left\{\vec{\Theta}_{1}, \vec{\Theta}_{k}^{(0)}, k=2, \ldots, 2 N\right\}$, which of course still fulfills (A.1) and (A.2). In the second case, define two new vectors $\vec{\Theta}_{1}$ and $\vec{\Theta}_{2}$ by

$$
\begin{aligned}
& \vec{\Theta}_{1}=\frac{1}{\sqrt{2(1+d)}}\left(e^{i \delta} \vec{\Theta}_{1}^{(0)}+e^{-i \delta} \vec{\Theta}_{1}^{(0)^{*}} \cdot C\right) \\
& \vec{\Theta}_{2}=\frac{i}{\sqrt{2(1-d)}}\left(e^{i \delta} \vec{\Theta}_{1}^{(0)}-e^{-i \delta} \vec{\Theta}_{1}^{(0)}{ }^{*} \cdot C\right),
\end{aligned}
$$

where

$$
\left(\vec{\Theta}_{1}^{(0)^{*}} \cdot C\right) \cdot \vec{\Theta}_{1}^{(0)}=d e^{2 i \delta}
$$

with $d \in[0,1)$ and $\delta \in[0, \pi)$. Now choose $2 N-2$ new vectors $\vec{\Theta}_{k}^{(1)}$ to be orthonormal eigenvectors of $M$ perpendicular to the subspace spanned by $\vec{\Theta}_{1}$ and $\vec{\Theta}_{2}$ such that the two sets $\mathcal{B}^{(0)}$ and $\mathcal{B}^{(1)}, \mathcal{B}^{(1)}=\left\{\vec{\Theta}_{1}, \vec{\Theta}_{2}, \vec{\Theta}_{k}^{(1)}, k=3, \ldots, 2 N\right\}$, span the same space (one can obviously restrict this orthogonalization procedure to the set of vectors $\vec{\Theta}_{k}^{(0)}$ that span the eigenspace of $M$ belonging to the eigenvalue $\Lambda_{1}$ ). This ensures that the new set $\mathcal{B}^{(1)}$ fulfills the relations (A.1) and (A.2) and, in addition,

$$
\vec{\Theta}_{j}^{*} \cdot C=\vec{\Theta}_{j} \quad, \quad j \in\{1,2\} .
$$

By iteration of this process (that means performing successive changes of basis where in each step one or two additional vectors comply with Eq. (A.3)), one finally - after at most $2 N$ steps - ends up with a set $\mathcal{B}$ that fulfills the proposition. 


\section{Appendix B}

This Appendix contains another proof that has been omitted in Sec. 3 in order to keep the representation easier to survey. It shall be shown that all eigenvalues of the matrix $\widehat{A}(\alpha, \phi)$ (3.19) are at least doubly degenerate (in fact the degree of degeneration is always even). Even more, if $\widehat{U}$ (3.23) is any unitary transformation compatible with the condition (3.26) that diagonalizes $\widehat{A}(\alpha, \phi)$ for some fixed values of the parameters, then the diagonal matrix $\widehat{A}_{D}=\widehat{U}^{\dagger} \widehat{A}(\alpha, \phi) \widehat{U}$ has the form

$$
\widehat{A}_{D}=\left(\begin{array}{cc}
\Lambda^{2} & \mathbf{0}_{N} \\
\mathbf{0}_{N} & \Lambda^{2}
\end{array}\right),
$$

where $\Lambda$ denotes the diagonal $N \times N$ matrix with elements $\Lambda_{k}, k=1, \ldots, N$.

The proof of this fact will be organized as follows. First, we will take into account that the Bogoliubov transformation (3.9) is of course not uniquely determined by the claimed properties. From this consideration, it will be possible to find two different transformations that yield the same diagonal matrix $\widehat{A}_{D}$ and the conjectured form of $\widehat{A}_{D}$ follows directly. As will be seen in what follows, the real cause of the degeneration lies in the fact that by exchanging the role of creation and annihilation operators one changes the sign of the $\Lambda_{k}$.

Assume that one succeeded to find a certain unitary transformation $\widehat{U}$ that diagonalizes $\widehat{A}(\alpha, \phi)$ for definite values of $\alpha$ and $\phi$ and in addition complies with the condition (3.26). It was proved in Appendix A that this is always possible. Then you have

$$
\widehat{A}_{D}(\alpha, \phi)=\widehat{U}^{\dagger} \widehat{A}(\alpha, \phi) \widehat{U}=\left(\begin{array}{cc}
\Lambda^{2} & \mathbf{0}_{N} \\
\mathbf{0}_{N} & \Lambda^{\prime 2}
\end{array}\right),
$$

where now $\Lambda$ and $\Lambda^{\prime}$ are diagonal $N \times N$ matrices with elements $\Lambda_{k}$ and $\Lambda_{k}^{\prime}$, respectively $(k=1, \ldots, N)$. Now, suppose you perform a Bogoliubov transformation (3.9) involving only creation and annihilation operators $a_{j}^{\dagger}$ and $a_{j}$ with one fixed index $j$, that is

$$
\begin{aligned}
& \tilde{a}_{j}=g a_{j}+h^{*} a_{j}^{\dagger} \\
& \tilde{a}_{j}^{\dagger}=h a_{j}+g^{*} a_{j}^{\dagger},
\end{aligned}
$$

where the $2 \times 2$ matrix $u$ defined by

$$
u=\left(\begin{array}{cc}
g & h^{*} \\
h & g^{*}
\end{array}\right)
$$

is unitary (note that this implies that either $g$ or $h$ is equal to zero and that the determinant $\operatorname{det}(u) \in\{1,-1\})$. This transformation will not alter the anticommutation relations, thus the operators $\tilde{a}_{j}^{\dagger}$ and $\tilde{a}_{j}$ are fermionic operators as well. On the other hand, the Hamiltonian $\tilde{H}$ (3.8) given by

$$
\tilde{H}=\sum_{k=1}^{N} \Lambda_{k} a_{k}^{\dagger} a_{k}+E_{0}
$$

is transformed into

$$
\tilde{H}=\sum_{k=1}^{N}\left(1-\delta_{j, k}\right) \Lambda_{k} a_{k}^{\dagger} a_{k}+\operatorname{det}(u) \Lambda_{j} \tilde{a}_{j}^{\dagger} \tilde{a}_{j}+\Lambda_{j} h h^{*}+E_{0} .
$$

This means that if $\operatorname{det}(u)=1$ the Hamiltonian is not changed (since this implies $h=0$ ), whereas in the case $\operatorname{det}(u)=-1$ the sign of $\Lambda_{j}$ is altered and the ground-state energy is shifted by the amount $\Lambda_{j} h h^{*}=\Lambda_{j}$. Of course one can perform a transformation as such independently for every value of $k, k=1, \ldots, N$.

It follows that the equations deduced from the necessary conditions (3.13)

$$
\left[a_{k}, \tilde{H}\right]=\Lambda_{k} a_{k}
$$


are - up to the signs of the $\Lambda_{k}$ - identical, independent of any performed transformation of the above-mentioned type. The shift in the constant term $E_{0}$ (see Eqs. (B.4) and (B.5) drops out because of the use of the commutator in Eq. (B.6). However, in the final eigenvalue equations (3.18) only the squares of the $\Lambda_{k}$ enter the calculation, therefore these are not altered at all. This leads to the consequence that the diagonal matrix $\widehat{A}_{D}$ is invariant under any of these transformations (B.3), where of course one has to take into account the change of basis performed in the derivation of the eigenvalue equation. In other words, the whole problem has a $U(1)^{\otimes N}$-invariance corresponding to an arbitrary phase factor for each pair of creation and annihilation operators. But the eigenvalue equations (3.18) beyond this are not altered by an exchange of creation and annihilation operators, therefore they enjoy an $\left(U(1) \otimes_{s} \mathbf{Z}_{2}\right)^{\otimes N}$-invariance, where $\otimes_{s}$ denotes a semi-direct product with the normal divisor (invariant subgroup) on the left side.

Now, take as a special transformation of this form the matrix $\widehat{C}$ defined by

$$
\widehat{C}=\left(\begin{array}{cc}
\mathbf{0}_{N} & -i \mathbf{1}_{N} \\
i \mathbf{1}_{N} & \mathbf{0}_{N}
\end{array}\right)
$$

This acts on the $a_{k}^{\dagger}$ and $a_{k}$ in the same way for all $k$, namely through

$$
\begin{aligned}
& a_{k} \longmapsto \tilde{a}_{k}=i a_{k}^{\dagger} \\
& a_{k}^{\dagger} \longmapsto \tilde{a}_{k}^{\dagger}=-i a_{k} .
\end{aligned}
$$

It follows that the transformation $V$ that is related to $\widehat{U}=T U$ (see Eqs. (3.24) and (3.23) by

$$
V=T \widehat{C} U=C T U=C \widehat{U}=C \widehat{U}^{*} C
$$

with

$$
C=\left(\begin{array}{cc}
\mathbf{0}_{N} & \mathbf{1}_{N} \\
\mathbf{1}_{N} & \mathbf{0}_{N}
\end{array}\right) \quad, \quad T=\frac{1}{\sqrt{2}}\left(\begin{array}{cc}
\mathbf{1}_{N} & \mathbf{1}_{N} \\
i \mathbf{1}_{N} & -i \mathbf{1}_{N}
\end{array}\right)
$$

diagonalizes $\widehat{A}$ to the same diagonal matrix $\widehat{A}_{D}$ as $\widehat{U}$ does. That means that $\widehat{A}_{D}$ has to commute with $C$ and, using Eq. (B.2), one therefore obtains the result

$$
\Lambda^{2}=\Lambda^{\prime 2}
$$

that proves the assertion (B.1).

\section{References}

[1] E. Ising, Z. Phys. 21 (1925) 613.

[2] J. B. Kogut, Rev. Mod. Phys. 51 (1979) 659.

[3] R. J. Baxter, Exactly Solved Models in Statistical Mechanics. Acad. Press, 1982.

[4] L. Onsager, Phys. Rev. 65 (1944) 117.

[5] E. Lieb, T. Schultz and D. Mattis, Ann. Phys. 16 (1961) 407.

[6] A. A. Belavin, A. M. Polyakov and A. B. Zamolodchikov, Nucl. Phys. B 241 (1984) 333.

[7] D. Friedan, Z. Qiu and S. Shenker, Phys. Rev. Lett. 52 (1984) 1575.

[8] J. L. Cardy, Nucl. Phys. B 240 (1984) 514, Nucl. Phys. B 275 (1986) 200.

[9] L. Turban, J. Phys. A: Math. Gen. 18 (1985) L325. 
[10] M. Henkel and A. Patkós, Nucl. Phys. B 285 [FS 19] (1987) 29, J. Phys. A: Math. Gen. 20 (1987) 2199, J. Phys. A: Math. Gen. 21 (1988) L231.

[11] M. Henkel, A. Patkós and M. Schlottmann, Nucl. Phys. B 314 (1989) 609.

[12] M. Baake, P. Chaselon and M. Schlottmann, Nucl. Phys. B 314 (1989) 625.

[13] M. Schlottmann, Diploma thesis, Bonn-IR-88-41 (1988).

[14] M. Baake, P. Christe and V. Rittenberg, Nucl. Phys. B 300 [FS22] (1988) 637.

[15] H. Hinrichsen, Preprint Bonn-HE-89-12 (1989).

[16] T. W. Burkhardt and I. Guim, J. Phys. A: Math. Gen. 18 (1985) L33, Phys. Rev. B 35 (1987) 1799.

[17] G. v. Gehlen and V. Rittenberg, J. Phys. A: Math. Gen. 19 (1986) L631.

[18] P. Chaselon, J. Phys. A: Math. Gen. 22 (1989) 2495.

[19] M. Baake., J. Math. Phys. 29 (1988) 1753.

[20] A. Rocha-Caridi, in Vertex Operators in Mathematics and Physics, ed. J. Lepowski, S. Mandelstam and I. Singer. Springer, New York, 1985.

[21] M. Baake, Ph.D. thesis, Bonn-IR-87-09 (1987).

[22] H. W. Blöte, J. L. Cardy and M. P. Nightingale, Phys. Rev. Lett. 56 (1986) 742.

[23] I. Affleck, Phys. Rev. Lett. 56 (1986) 746.

[24] P. Goddard and D. Olive, Int. J. Mod. Phys. A 1 (1986) 303.

[25] C. Lanczos, J. Res. Nat. Bur. Stand. 45 (1950) 225.

[26] R. Bulirsch and J. Stoer, Num. Math. 6 (1964) 413.

[27] M. Henkel and G. Schütz, J. Phys. A: Math. Gen. 21 (1988) 2617.

[28] T. Wittlich, Preprint Bonn-HE-89-06 (1989), Diploma thesis, Bonn-IR-89-34 (1989).

[29] U. Grimm, Diploma thesis, Bonn-IR-88-30 (1988). 